\title{
Assessment of the Relationship of Anthropometric Indices With Vitamin D, Zinc, and Fasting Blood Sugar Levels in Women With and Without Infection Following Abdominal Hysterectomy: A Case-Control Study
}

\author{
Sanaz Mousavi ${ }^{1}$, Hamid Owaysee Osquee ${ }^{1}$, Seyed Mehdi Haghdoost ${ }^{1 *}$
}

\begin{abstract}
Objectives: It is necessary to prevent postoperative infection following abdominal hysterectomy. The present study aimed to assess the relationship of anthropometric indices with vitamin D, zinc (Zn), and fasting blood sugar (FBS) levels in women with and without infection following abdominal hysterectomy in order to determine the exact effects of these factors on infection.

Materials and Methods: This case-control study was conducted in three teaching hospitals affiliated with Tabriz University of Medical Sciences in an 18-month period leading up to 2020. The participants consisted of 90 women who were candidates for abdominal hysterectomy. Anthropometric indices and vitamin D, Zn, and FBS levels were measured before the surgery. These factors were compared in the two groups of women with and without infection using the independent t-test and Pearson correlation coefficient, and $P<0.05$ was statistically significant.

Results: No significant relationship was found between vitamin D, Zn, and FBS levels and anthropometric indices except for body mass index (BMI) that had a significant negative relationship with vitamin D blood levels $(r=-0.20$ and $P=0.043)$ while a significant positive relationship with FBS levels $(r=+30$ and $P=0.036)$ in infected patients. Therefore, patients with a higher BMI showed lower vitamin D whereas higher FBS levels in the group with infection.

Conclusions: In general, vitamin D levels decreased and FBS levels increased by an increase in the BMI. These factors altogether increased the risk of postoperative infection following abdominal hysterectomy.

Keywords: Abdominal Hysterectomy, Infection, Zinc, Vitamin D
\end{abstract}

\section{Introduction}

Hysterectomy is the second most common surgical procedure performed in women and its prevalence varies in different populations. It can be performed either abdominally or laparoscopically $(1,2)$. Infection is one of the relatively common postoperative complications in abdominal hysterectomy, and its reported incidence rate is higher than $8 \%$ (3). However, no reliable reports are available on its prevalence (4). Postoperative infection following abdominal hysterectomy affects the positive outcomes of surgery and leads to a wide range of complications. Therefore, it is important to prevent infection and determine its risk factors in order to prevent the occurrence of postoperative infection following abdominal hysterectomy (5-8).

Many factors have been reported to influence postoperative infection, including vitamins and minerals. One study reported that vitamin $\mathrm{D}$ deficiency led to infection (9). In addition, it has somehow been proved that zinc $(\mathrm{Zn})$ enhances and accelerates recovery after surgery (10). Diabetes and hyperglycemia are the strongest risk factors for infection in women without autoimmune diseases (11). All the above-mentioned variables might be associated with anthropic indices. However, the extent and intensity of the relationships are not accurately known yet (12).

The incidence of postoperative infection following abdominal hysterectomy is relatively high and the infection has irreparable side effects on the outcomes of the surgery. Further, vitamin D, Zn, and blood glucose levels have possible effects on the risk of infections. Given the undetermined relationships of the above-mentioned parameters with anthropometric indices, the present study sought to evaluate the relationship of anthropometric indices with vitamin $\mathrm{D}, \mathrm{Zn}$, and FBS levels in women with and without postoperative infection following abdominal hysterectomy. The obtained results can be considered in making plans for the prevention of postoperative infection after abdominal hysterectomy.

\section{Materials and Methods}

The present case-control study was conducted in AlZahra, Imam Reza, and Taleghani hospitals affiliated to Tabriz University of Medical Sciences in an 18-month 
Key Messages

- Vitamin D, zinc and FBS can play a role in posthysterectomy infections.

- High body mass index decreases vitamin D levels and increases FBS.

- Decreased vitamin D levels and increased FBS increase the risk of infection from hysterectomy.

period leading up to 2020. The sample size $(n=85)$ was determined by taking into account the results of similar studies $(4,12)$, along with a statistical power of $80 \%$, a confidence level of $95 \%$, and a correlation coefficient of 0.3 . The final sample size $(n=90)$ was selected to offset sample attritions. The number of participants was the same in all three hospitals since equal numbers of patients were referred to these medical centers for hysterectomy. Ac convenient sampling technique was used to select the participants. The inclusion criteria were candidates for abdominal hysterectomy, $>30$ years of age, and a willingness to participate in the study. On the other hand, the exclusion criteria included a history of vitamin $\mathrm{D}$ and Zn intake in the past three months, affliction with diabetes mellitus for more than 5 years, a malignancy, history of systemic infectious diseases, tobacco and alcohol consumption, opiate addiction, and continuous antibiotic intake during the past week. The study groups carefully followed the inclusion and exclusion criteria of the study, and the main purpose of the study was the outcome of this study. The patients were then divided into two groups with and without infection.

Diagnostic criteria for infection were laboratory tests (i.e., erythrocyte sedimentation rate, white blood cell, and C-reactive protein), swelling, redness, itching, and wound exudate at the incision site when the participants were examined by two infectious disease specialists (one of whom was a member of the research team). Those diagnosed as infectious patients according to the abovementioned criteria were and healthy patients included in the infectious patients and control groups, respectively. A checklist on patients' personal information was completed before the surgery, including age, height, weight, body mass index (BMI), waist circumference, hip circumference, and the waist-to-hip ratio (weight was measured using the adult weighing scale ZT-120 with an accuracy rate of $0.5 \mathrm{~kg}$, and height was measured with a meter attached to the scale with an accuracy rate of $1 \mathrm{~cm}$ ). Vitamin D) by the ELISA CLIA method in the laboratory of Imam Reza Hospital(, $\mathrm{Zn}$ (the blood clot was centrifuged to separate the serum from the cell fraction and then evaluated by atomic absorption spectrophotometry), and FBS (fasting blood sugar, blood samples were evaluated in the laboratory by the enzymatic method of glucose oxidase) levels were measured in the hospital on the day before the surgery. Five $\mathrm{mL}$ of the blood was taken from the brachial vein by a skilled nurse who was not a member of the research team). The samples were transferred to the laboratory. All mentioned criteria were carefully measured for each patient in each group. The normal values of vitamin D, $\mathrm{Zn}$, and FBS are listed in Table 1 (Figure 1).

The project was approved by the Ethics Committee of Tabriz University of Medical Sciences (Code of Ethics: IR.TBZMED.REC.1398.736). The researchers announced the research project in the hospitals to find patients who were willing to participate in the study. The objectives of the research were explained to those who agreed to participate in the research in as simple terms as possible. Written informed consent forms were collected from them, and ethical considerations were also observed as in other studies related to infectious diseases (13-15).

Data were completed and collected by the researcher. SPSS statistical software (version 20) was used after checking the validity of all questionnaires by two statistical consultants. Then, the obtained data were analyzed using the Pearson correlation coefficient (the correlations of anthropometric indices with vitamin $\mathrm{D}, \mathrm{Zn}$, and FBS levels), the independent $t$ test (Comparing all variables between the two groups), and one-way ANOVA test (evaluating the relationship between BMI and vitamin $\mathrm{D}$ and FBS levels in the infected patients) at a $P<0.05$ significance level.

\section{Results}

In general, 149 patients visited the hospitals during the mentioned period for abdominal hysterectomy, and 90 eligible patients participated in the study. The mean and standard deviation of age, weight, and BMI of the participants were $45.89 \pm 5.29,63.59 \pm 10.93$, and $27.78 \pm 3.55$, respectively. Both groups were matched in terms of variables other than the ones assessed in this study. Demographic data, anthropometric indices, vitamin $\mathrm{D}, \mathrm{Zn}$, and FBS levels of the patients are provided in Table 2.

Based on the assessment of the relationship of waist circumference, hip circumference, BMI, height, and weight with vitamin $\mathrm{D}, \mathrm{Zn}$, and FBS levels in women with and without infection, BMI had a significant negative relationship with FBS $(\mathrm{R}=+30, P=0.036)$ in patients with infection with the blood levels of vitamin $\mathrm{D}(\mathrm{R}=-0.20$, $P=0.043)$ and the remaining relationships were non-

Table 1. Normal Levels of Vitamin D, Zinc, and FBS

\begin{tabular}{ll}
\hline Test Name & Scope of Measurement \\
\hline \multirow{3}{*}{ Vitamin D } & Shortage: $10 \mathrm{ng} / \mathrm{dL}$ \\
& Insufficient: $10-30 \mathrm{ng} / \mathrm{dL}$ \\
& Enough: $30-100 \mathrm{ng} / \mathrm{dL}$ \\
& Shortage $<70 \mu \mathrm{g} / \mathrm{dL}$ \\
Zinc & Normal: $70-120 \mu \mathrm{g} / \mathrm{dL}$ \\
& Above normal $>120 \mu \mathrm{g} / \mathrm{dL}$ \\
& Normal: $60-90$ \\
FBS & Abnormal: $>90 \mathrm{mg} / \mathrm{dL}$ \\
\hline
\end{tabular}

Note. FBS, Fasting blood sugar. 


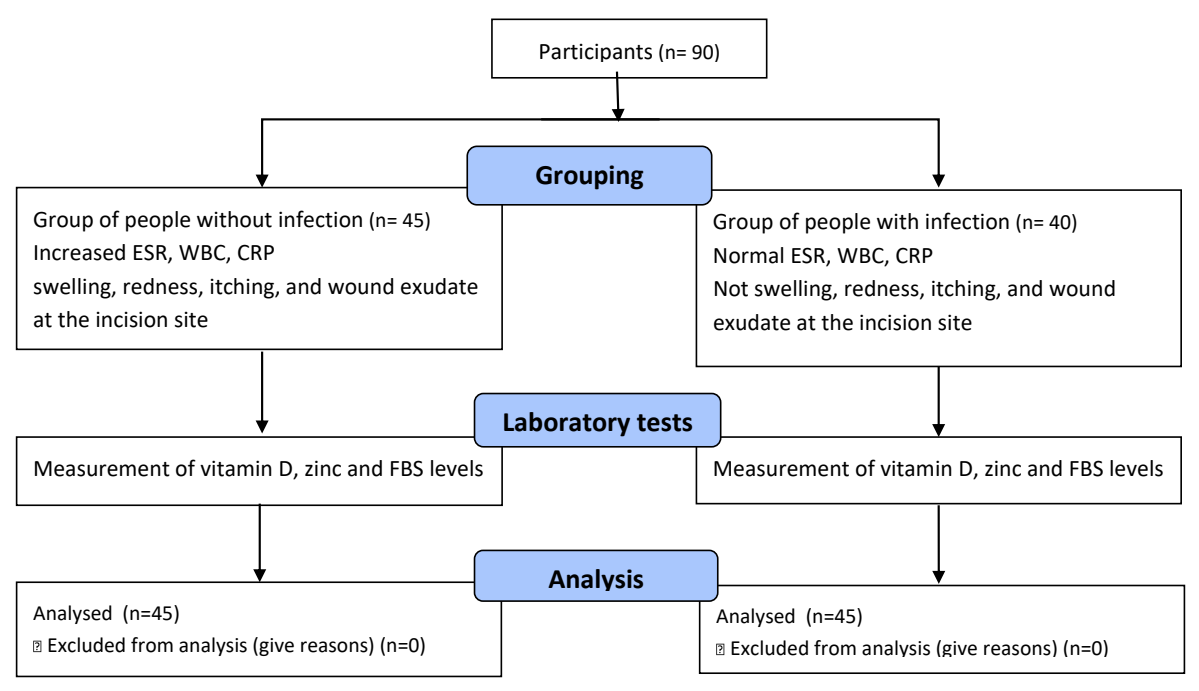

Figure 1. Flowchart Examining the Laboratory Tests of Study Participants.

significant $(P>0.05)$. Related information is summarized in detail in Table 3.

Considering that the BMI in women with infection was significantly correlated with vitamin D and FBS, the relationship between these two variables with BMI scores was examined as well. The assessment of the BMI in the infected patients with vitamin D and FBS levels showed that patients with a normal BMI had normal vitamin D and FBS levels. Vitamin D levels decreased while FBS levels increased by an increase in the BMI in the infected patients (Table 4).

\section{Discussion}

The study aimed to assess the relationship between anthropometric indices and vitamin $\mathrm{D}, \mathrm{Zn}$, and FBS levels in women with and without infection following abdominal hysterectomy. The results showed that only vitamin D and FBS were significantly associated with BMI

Table 2. Demographic Data and Anthropometric Indices of the Participants

\begin{tabular}{|c|c|c|c|}
\hline Variable & Women With Infection $(n=45)$ & Women Without Infection $(n=45)$ & $P$ Value $^{a}$ \\
\hline Age (y) & $44.15 \pm 5.44$ & $45.89 \pm 5.12$ & 0.219 \\
\hline Weight (kg) & $7219 \pm 8.49$ & $71.77 \pm 10.03$ & 0.310 \\
\hline Height $(\mathrm{cm})$ & $159.41 \pm 11.18$ & $161.15 \pm 10.25$ & 0.119 \\
\hline $\mathrm{BMI}$ & $27.51 \pm 4.36$ & $26.40 \pm 5.03$ & 0.219 \\
\hline Waist circumference $(\mathrm{cm})$ & $88.19 \pm 8.43$ & $87.29 \pm 10.12$ & 0.119 \\
\hline Hip circumference (cm) & $10.81 \pm 15.41$ & $100.15 \pm 15.66$ & 0.102 \\
\hline Waist-to-hip ratio & $0.81 \pm 0.10$ & $0.80 \pm 0.11$ & 0.400 \\
\hline Vitamin D & $8.19 \pm 3.29$ & $49.19 \pm 5.49$ & 0.001 \\
\hline Zinc & $73.15 \pm 9.15$ & $121.44 \pm 15.76$ & 0.001 \\
\hline FBS & $95.49 \pm 9.59$ & $78.19 \pm 7.39$ & 009.0 \\
\hline
\end{tabular}

Note. BMI: Body mass index; FBS: Fasting blood sugar. ${ }^{a}$ Independent $t$ test.

Table 3. Relationships Between Anthropometric Indices and Zinc, Vitamin D, and FBS Levels in the Patients

\begin{tabular}{|c|c|c|c|c|c|c|}
\hline & & Waist circumference $(\mathrm{cm})$ & Hip circumference $(\mathrm{cm})$ & BMI & Height $(\mathrm{cm})$ & Weight (kg) \\
\hline \multirow{4}{*}{ Vitamin D } & Women with infection & $R=+0.17$ & $\mathrm{R}=+0.21$ & $R=-0.20$ & $R=+0.18$ & $\mathrm{R}=+0.17$ \\
\hline & $(n=45)$ & $P=0.215$ & $P=0.081$ & $P=0.043$ & $P=0.401$ & $P=0.201$ \\
\hline & Women without & $R=+0.15$ & $R=+0.15$ & $R=+0.18$ & $\mathrm{R}=+0.14$ & $\mathrm{R}=+0.15$ \\
\hline & infection $(n=45)$ & $P=0.503$ & $P=0.208$ & $P=0.303$ & $P=0.501$ & $P=0.201$ \\
\hline \multirow{4}{*}{ Zinc } & Women with infection & $R=+0.21$ & $R=+0.20$ & $R=+0.20$ & $R=+0.20$ & $\mathrm{R}=+0.21$ \\
\hline & $(\mathrm{n}=45)$ & $P=0.311$ & $P=0.219$ & $P=0.203$ & $P=0.189$ & $P=0.201$ \\
\hline & Women without & $R=+0.21$ & $R=+0.21$ & $R=+0.20$ & $\mathrm{R}=+0.20$ & $R=+0.19$ \\
\hline & infection $(n=45)$ & $P=0.303$ & $P=0.288$ & $P=0.251$ & $P=0.209$ & $P=0.209$ \\
\hline \multirow{4}{*}{ FBS } & Women with infection & $R=+0.15$ & $R=+0.19$ & $R=+0.30$ & $R=+0.15$ & $R=+0.21$ \\
\hline & $(n=45)$ & $P=0.312$ & $P=0.209$ & $P=0.036$ & $P=0.199$ & $P=0.198$ \\
\hline & Women & $R=+0.18$ & $R=+0.19$ & $R=+0.19$ & $R=+0.18$ & $R=+0.17$ \\
\hline & infection $(n=45)$ & $P=0.309$ & $P=0.341$ & $P=0.291$ & $P=0.258$ & $P=0.213$ \\
\hline
\end{tabular}

Note. BMI: Body mass index; FBS: Fasting blood sugar. Pearson test was used for analysis data. 
Table 4. Relationship Between BMI and Vitamin D and FBS Levels in the Infected Patients

\begin{tabular}{lcc}
\hline BMI & Vitamin D & FBS \\
\hline Normal & $16.19 \pm 5.21$ & $91.41 \pm 9.29$ \\
Overweight & $9.25 \pm 2.03$ & $111.14 \pm 10.83$ \\
Obese & $3.14 \pm 1.41$ & $129.56 \pm 15.41$ \\
$P$ value $^{\mathrm{a}}$ & 0.003 & 0.009 \\
\hline
\end{tabular}

Note. FBS: Fasting blood sugar; BMI: Body mass index; ANOVA:

a One-way analysis of variance (ANOVA).

in women with infection. In other words, the BMI had a significant positive relationship with vitamin $\mathrm{D}$ whereas a significant negative relationship with FBS. Recent studies have demonstrated that the blood levels of minerals and vitamins (e.g., Zn, vitamin D, and FBS) in anthropometric indices could increase the risk of postoperative infection (16-19).

The results of this study also indicated a significant negative correlation between the BMI and the blood levels of vitamin $\mathrm{D}$ in patients with infection. In other words, vitamin D levels decreased in patients with a higher BMI who developed an infection after abdominal hysterectomy surgery. Obese patients were more prone to postoperative infection compared to those with a normal BMI. Therefore, there was an inverse relationship between these two variables. Low levels of vitamin D levels and high levels of BMI were risk factors for postoperative infection. These results are consistent with those of Patil et al (20) and Göksever Çelik et al (21).

Based on the results, FBS had a positive significant correlation with BMI in women with infection. FBS levels increased in obese people, which led to infection following hysterectomy. High blood sugar levels (hyperglycemia) are a risk factor for postoperative infection. Some studies reported an increased risk of postoperative infection in patients with higher FBS levels. Prominent studies confirmed the high prevalence of infection in patients with diabetes compared to those without diabetes. Therefore, it is necessary to measure and control FBS levels in those undergoing surgery. These results are in line with the findings of Corrigan et al (22), Jiamset et al (23), and Fakhari et al (11).

A high BMI is a risk factor for postoperative infection. The blood levels of vitamin D decrease in people with a higher BMI, which can dramatically increase the risk of postoperative infection. However, the FBS level increases in those with a high BMI, leading to an increase in the risk of postoperative infection. IIt seems that the combination of the mentioned variables has cumulative additive effects and increases the risk of postoperative infection following abdominal hysterectomy. Also, other studies in the field of medical sciences indicate the effects of vitamins on infection, so that their levels are better considered (24-28).

\section{Limitations}

The lack of taking into account the reasons for performing a hysterectomy, the lack of information on the applied hysterectomy techniques, and the time of surgery were among the limitations and weaknesses of the present study.

\section{Suggestions for Future Studies}

The authors recommend more research should be carried out while removing the limitations of this study. There are interventions such as the administration of supplements containing vitamin $\mathrm{D}$ and $\mathrm{Zn}$, and obese patients are encouraged to lose weight before hysterectomy.

\section{Conclusions}

Postoperative infection following hysterectomy depends on many factors, and the identification of these factors can help in making effective healthcare-associated plans. Among the strongest factors is the BMI, which decreases vitamin D levels while increasing FBS levels. These factors altogether increase the risk of postoperative infection following abdominal hysterectomy.

\section{Authors' Contribution}

SM: Study design, data review, manuscript writing; HOO: data collection, data review, manuscript writing; $\mathrm{SMH}$ : manuscript writing, article submission and response to comments.

Conflict of Interests

The authors declared no conflict of interests.

Financial Support

This study was financially supported by the Ethics Committee of Tabriz University of Medical Sciences and the ethics number indicates financial support for this project.

\section{Acknowledgments}

This study is the result of a small portion of Ms. Solmaz Sayed's General Practitioner course. The researchers are grateful to Ms. Solmaz Sayedin for collecting information, as well as patients for participating in the study, and the Vice-President of Research and Technology of Tabriz University of Medical Sciences for financial support.

\section{References}

1. Khanbabaei Gol M, Aghamohamadi D. Effect of intravenous infusion of magnesium sulfate on opioid use and hemodynamic status after hysterectomy: double-blind clinical trial. Iran J Obstet Gynecol Infertil. 2019;22(7):32-38. doi:10.22038/ijogi.2019.13815

2. Egbe TO, Kobenge MF, Arlette MM, Egbe EN, Nyemb JE, Mbu RE. Prevalence and outcome of hysterectomy at the Douala General Hospital, Cameroon: a cross-sectional study. Int J Surg Res Pract. 2018;5(4):092. doi:10.23937/2378-3397/1410092

3. Carrubba AR, Whitmore GT, Radhakrishnan SJ, Sheeder J, Muffly TM. Postoperative infections in women undergoing hysterectomy for benign indications: a cohort study. Minerva Ginecol. 2019;71(4):263-271. doi:10.23736/s0026-4784.19.04365-x

4. Yazdani SH, Bouzari Z, Abedi Samakoosh M, Nazari A, Daryabari A. Risk factors incidence of surgical wound infection following abdominal hysterectomy. J Gorgan Univ Med Sci. 2014;15(4):90-95. [Persian].

5. Morgan DM, Swenson CW, Streifel KM, et al. Surgical site infection following hysterectomy: adjusted rankings in a regional collaborative. Am J Obstet Gynecol. 2016;214(2):259.e1-259.e8. doi:10.1016/j. ajog.2015.10.002

6. Steiner HL, Strand EA. Surgical-site infection in gynecologic surgery: pathophysiology and prevention. Am J Obstet Gynecol. 2017;217(2):121-128. doi:10.1016/j.ajog.2017.02.014 
7. Aghamohamadi D, Mehdinavaz Aghdam A, Khanbabayi Gol M. Prevalence of infections associated with port and predisposing factors in women with common cancers under chemotherapy referred to hospitals in Tabriz in 2015. Iran J Obstet Gynecol Infertil. 2019;21(11):7-13. doi:10.22038/ijogi.2019.12321

8. Solomayer EF, Radosa JC, Juhasz-Böss I, Mavrova R. Abdominal hysterectomy: postoperative care. In: Alkatout I, Mettler L, eds. Hysterectomy: A Comprehensive Surgical Approach. Cham: Springer; 2018:1347-1351. doi:10.1007/978-3-319-22497-8_109

9. Ghorbani Abdehgah A, Monshizadeh A, Mohajeri Tehrani M, et al. Relationship between preoperative 25-hydroxy vitamin $\mathrm{D}$ and surgical site infection. J Surg Res. 2020;245:338-343. doi:10.1016/j. jss.2019.07.036

10. Abdullah BJ, Atasoy N, Omer AK. Evaluate the effects of platelet rich plasma (PRP) and zinc oxide ointment on skin wound healing. Ann Med Surg (Lond). 2019;37:30-37. doi:10.1016/j.amsu.2018.11.009

11. Fakhari S, Bile Jani I, Atashkhouei S, Khanbabayi Gol M, Soliemanzadeh S. Comparing the effect of hypotension treatment due to spinal anesthesia with ephedrine or phenylephrine on arterial blood gases and neonatal Apgar score during cesarean delivery in obese mothers: randomized clinical trial. The Iranian Journal of Obstetrics, Gynecology and Infertility. 2019;22(10):12-20. doi:10.22038/ijogi.2019.14185

12. Hosseini-Fard R, Khosravi M, Shamseddin Beiranvand A, et al. Correlation of PCSK9 and serum sdLDL levels and other demographic and laboratory indicators in healthy individuals. Qom Univ Med Sci J. 2018;12(3):36-45. doi:10.29252/qums.12.3.36

13. Haghdoost M, Mousavi S, Khanbabayi Gol M, Montazer M. Frequency of Chlamydia trachomatis infection in spontaneous abortion of infertile women during first pregnancy referred to Tabriz University of Medical Sciences by nested PCR method in 2015. Int J Womens Health Reprod Sci. 2019;7(4):526-530. doi:10.15296/ijwhr.2019.87

14. Taghizadeh S, Haghdoost M, Mashrabi O, Zeynalikhasraghi Z. Antibiotic usage in intensive care units of Tabriz Imam Reza hospital, 2011. Am J Infect Dis. 2013;9(4):123-8.

15. Akbari Aghdam $M$, Soroush Barhaghi $\mathbf{M H}$, Aghazadeh $M$, et al. Virulence genes in biofilm producer Enterococcus faecalis isolates from root canal infections. Cell Mol Biol (Noisy-le-grand). 2017;63(5):5559. doi:10.14715/cmb/2017.63.5.11

16. Turan A, Artis AS, Hanline C, et al. Preoperative vitamin D concentration and cardiac, renal, and infectious morbidity after noncardiac surgery. Anesthesiology. 2020;132(1):121-130. doi:10.1097/ aln.0000000000003000

17. Federico G, Genoni A, Puggioni A, et al. Vitamin D status, enterovirus infection, and type 1 diabetes in Italian children/adolescents. Pediatr Diabetes. 2018;19(5):923-929. doi:10.1111/pedi.12673

18. Gomes TL, Fernandes RC, Vieira LL, et al. Low vitamin D at ICU admission is associated with cancer, infections, acute respiratory insufficiency, and liver failure. Nutrition. 2019;60:235-240. doi:10.1016/j.nut.2018.10.018

19. Bischoff-Ferrari HA, Bhasin S, Manson JE. Preventing fractures and falls: a limited role for calcium and vitamin D supplements? JAMA. 2018;319(15):1552-1553. doi:10.1001/jama.2018.4023

20. Patil MB, Ragav ED. A clinical, biochemical profile of type-2 diabetes in women with special reference to vitamin-D status in obese and nonobese. J Assoc Physicians India. 2018;66(12):21-24.

21. Göksever Çelik H, Çelik E, Turan G, Seçkin KD, Gedikbaşı A. Risk factors for surgical site infection after hysterectomy. J Infect Dev Ctries. 2017;11(4):355-360. doi:10.3855/jidc.9053

22. Corrigan KE, Vargas MV, Robinson HN, et al. Impact of diabetes mellitus on postoperative complications following laparoscopic hysterectomy for benign indications. Gynecol Obstet Invest. 2019;84(6):583-590. doi:10.1159/000501034

23. Jiamset I, Hanprasertpong J. Impact of diabetes mellitus on oncological outcomes after radical hysterectomy for early stage cervical cancer. J Gynecol Oncol. 2016;27(3):e28. doi:10.3802/jgo.2016.27.e28

24. Sabeg PA, Mehrabi E, Nourizadeh R, Poursharifi H, Mousavi S. the effect of counseling on breast cancer awareness in rural Iranian women: a randomized controlled clinical trial. J Cancer Educ. 2019;34(6):10831091. doi: 10.1007/s13187-018-1411-z

25. Nikkhesal N, Nourizadeh R, Dastgiri S, Mehrabi E. The factors affecting women's decision about unplanned pregnancy: A hierarchical modeling strategy. Int J Womens Health Reprod Sci. 2018;6(4):483-90. doi:10.15296/ijwhr.2018.80

26. Nourizadeh R, Azami S, Farshbaf-Khalili A, Mehrabi E. The effect of motivational interviewing on women with overweight and obesity before conception. J Nutr Educ Behav. 2020;52(9):859-866. doi:10.1016/j.jneb.2020.04.219

27. Alizadeh-Sabeg P, Mehrabi E, Nourizadeh R, Hakimi S, Mousavi S. The effect of motivational interviewing on the change of breast cancer screening behaviors among rural Iranian women. Patient Educ Couns. 2021;104(2):369-374. doi:10.1016/j.pec.2020.08.011

28. Pourebrahim-Alamdari P, Mehrabi E, Nikkhesal N, Nourizadeh R, Esmaeilpour K, Mousavi S. Effectiveness of motivationally tailored interventions on cervical cancer screening: a systematic review and meta-analysis. Int J Womens Health Reprod Sci. 2021;9(2):86-90. doi: 10.15296/ijwhr.2021.16.

(c) 2021 The Author(s); This is an open-access article distributed under the terms of the Creative Commons Attribution License (http:// creativecommons.org/licenses/by/4.0), which permits unrestricted use, distribution, and reproduction in any medium, provided the original work is properly cited. 